\title{
GROWING HYDRODYNAMIC MODES IN KEPLERIAN ACCRETION DISKS DURING SECONDARY PERTURBATIONS: ELLIPTICAL VORTEX EFFECTS
}

\author{
Banibrata Mukhopadhyay ${ }^{1}$ \\ Received 2006 March 29; accepted 2006 August 17
}

\begin{abstract}
The origin of hydrodynamic turbulence, and in particular of an anomalously enhanced angular momentum transport, in accretion disks is still an unsolved problem. This is especially important for cold disk systems that are practically neutral in charge, since the turbulence in those systems cannot be of magnetohydrodynamic origin. While the flow must exhibit some instability and then turbulence in support of the transfer of mass inward and angular momentum outward, according to the linear perturbation theory, in the absence of magnetohydrodynamic effects, it should always be stable. We demonstrate that the three-dimensional secondary disturbance to the primarily perturbed disk, consisting of elliptical vortices, gives significantly large hydrodynamic growth in such a system and hence may suggest a transition to an ultimately turbulent state. This result is essentially applicable to accretion disks around quiescent cataclysmic variables, in protoplanetary and star-forming disks, and the outer region of disks in active galactic nuclei, where the gas is significantly colder, and thus the magnetic Reynolds number is smaller than $10^{4}$.
\end{abstract}

Subject headings: accretion, accretion disks — hydrodynamics — instabilities — turbulence

\section{INTRODUCTION}

The origin of turbulence and then the viscosity parameter $\alpha$, although it has been demonstrated for a hot Keplerian accretion disk by the magnetorotational instability (MRI; Balbus \& Hawley 1991; Balbus et al. 1996; Hawley et al. 1999), is still not well understood for cold disks, e.g., accretion disks around quiescent cataclysmic variables (Gammie \& Menou 1998; Menou 2000), protoplanetary and star-forming disks (Blaes \& Balbus 1994), and the outer region of disks in active galactic nuclei (Menou \& Quataert 2001), even after more than three decades of the famous discovery of the $\alpha$-disk (Shakura \& Sunyaev 1973). These cold systems are largely neutral in charge, so that the MRI-driven turbulence appears to be ruled out. However, to support accretion, some sort of turbulence must appear (most likely purely hydrodynamic in nature) and then the corresponding turbulent viscosity, as the molecular viscosity is negligible. Richard \& Zahn (1999) indeed showed by analyzing results of laboratory experiments for the classical Couette-Taylor flow that in the case where angular momentum increases outward, similar to the Keplerian flow, hydrodynamic turbulence may be sustained. Then they derived the corresponding turbulent viscosity, which is very useful for astrophysical purposes. Longaretti (2002) argued that the Keplerian accretion flow in the shearing-sheet limit should be turbulent and that the lack of turbulence in simulations is due to lack of resolution. Then Lesur \& Longaretti (2005) showed that it is not possible to achieve the required resolution with currently available computer resources. They also found that the efficiency of turbulent transport is directly correlated to the critical Reynolds number for the transition to turbulence. In spite of all these analyses, the actual origin of hydrodynamic turbulence in such systems still remains unclear. It is important to note that the numerical simulation has never been carried out with a very high Reynolds number, which is what the real accretion disk exhibits. The hydrodynamic effects may compete with the magnetohydrodynamic effects (essentially MRI) at a high Reynolds number, and therefore, in a realistic system, turbulence may be due to hydrodynamic effects independent of whether the disk is cold or hot.

Recently, several authors have put their efforts into making progress toward the solution of this difficult problem (e.g., Tevzadze et al. 2003; Chagelishvili et al. 2003; Umurhan \& Regev 2004, hereafter UR04; Yecko 2004; Mukhopadhyay et al. 2005, hereafter MAN05; Afshordi et al. 2005, hereafter AMN05; Johnson \& Gammie 2005; Umurhan et al. 2006; Barranco \& Marcus 2005; Papaloizou 2005a, 2005b). The main aim of these works is to demonstrate the pure hydrodynamic turbulence by transient growth of energy with the suitable choice of a two-dimensional initial perturbation. The idea is that any large growth could plausibly switch the accretion disk into the nonlinear regime, which might result in its subcritical transition to turbulence if energy growth exceeds the threshold for turbulence. One might argue that transient growth, even if large, cannot provide many clues to the existence and properties of a turbulent basin of attraction. Schmid \& Henningson (2001) and Criminale et al. (2003) have described in detail that the transition to turbulence is not a unique process and that it depends on the initial condition/disturbance and the nature of the flow. In fact, it is known that even in the presence of a secondary-instability linearly unstable base, flows may reach a nonturbulent saturated state. However, turbulence definitely belongs to the nonlinear regime, and it is exhibited only when large growth (or more precisely transient growth for the present purpose) switches the system over to the nonlinear regime. As our present goal is to understand the possible origin of hydrodynamic turbulence, we consider those situations in which large transient growth governs nonlinearity. In the present case where the accretion timescale is comparable to (or shorter than) the timescale needed to achieve maximum growth (MAN05; AMN05), the decaying nature of growth over a period longer than the accretion timescale does not matter. If growth (or maximum growth) becomes large enough that it exceeds the threshold to trigger nonlinearity and turbulence, then it does not matter whether growth evolves exponentially or transiently.

\footnotetext{
${ }^{1}$ Institute for Theory and Computation, Harvard-Smithsonian Center for Astrophysics, 60 Garden Street, MS-51, Cambridge, MA 02138; and Department of Physics, Indian Institute of Science, Bangalore-560012, India; bmukhopa@cfa.harvard.edu, bm@physics.iisc.ernet.in.
} 
Dauchot \& Manneville (1997) argued with a toy model that transient growth does not guarantee the transition to turbulence, as the underlying phase portraits do not get deformed much in the absence of a linearly unstable mode. However, that does not rule out the importance of transient growth, as their model is very simplistic, consisting of only two variables, and the growth is too tiny to expect any deformation in the phase portraits. Other alternative methods were proposed to describe the subcritical transition to turbulence and then to investigate various underlying aspects in detail by, e.g., Waleffe (1995, 1997), Brosa \& Grossmann (1999), Waleffe (2003), and Kerswell (2005), while the last two works, which discuss nonrotating Couette flows and pipe flows, respectively, are mostly relevant for astrophysical purposes. Although the works bring some new insight into the subject, the main problem, i.e., to understand the subcritical turbulence in three-dimensions, remains unsolved. This is indeed a nontrivial problem not only in astrophysics but also in fluid dynamics. In the present paper we suggest a possible mechanism that may govern the nonlinear effect in a Keplerian accretion disk. While our prescription does not solve the problem completely, it certainly opens up a new avenue to understanding the physics behind this puzzle.

The problem with transient growth, which has been proposed as the mechanism that generates turbulence (e.g., UR04; MAN05; AMN05), is that in two-dimensions, underlying perturbations must ultimately decline to zero in the viscous flow. To overcome this limitation, it is necessary to invoke three-dimensional effects. Various kinds of secondary instabilities, such as the elliptical instability, are widely discussed as a possible route to self-sustained turbulence in linearly perturbed shear flows (see, e.g., Pierrehumbert 1986; Bayly 1986; Hellberg \& Orszag 1988; Craik 1989; Le Diześ et al. 1996; Kerswell 2002). Therefore, we are motivated to see whether these three-dimensional instabilities are present in the Keplerian flows that consist of elliptical streamlines under two-dimensional perturbation. Goodman (1993) first explored the possible role of the elliptical instability in an accretion disk and Lubow et al. (1993) and Ryu \& Goodman (1994) showed that angular momentum may be transferred from the disk to the tidal source by the instability effect. They essentially considered the case of forced disks. Later, Ioannou \& Kakouris (2001) also examined transient growth being constantly reexcited by external noise.

The three-dimensional instability of a two-dimensional flow with elliptical vortices has been demonstrated by a number of authors (e.g., Craik \& Criminale 1986; Waleffe 1990) and has been proposed as a generic mechanism for the breakdown of many twodimensional high Reynolds number flows. This result motivates us to investigate whether similar scenarios exist in a Keplerian accretion disk. Therefore, we essentially plan to investigate the Keplerian flow with two consecutive perturbation effects. The primary twodimensional perturbation, which drives transient growth (MAN05; AMN05), governs elliptical streamlines in the flow. Then we consider the further three-dimensional perturbation (that is, the secondary one) to this two-dimensional flow to see if that drives the threedimensional instability effect in the presence of viscosity. Presumably, three-dimensional instabilities lead to nonlinear feedback and self-sustained turbulence.

Although the maximum growth in the Keplerian disk with finite vertical thickness and then with finite vertical perturbation is smaller than that in a two-dimensional Keplerian disk, vertical stratification may cause nonvanishing asymptotic growth that is a fraction of the maximum growth, as Tevzadze et al. (2003), MAN05, and AMN05 argued. However, they could not show whether this will significantly help the onset of turbulence. With three-dimensional hydrodynamic simulations, Barranco \& Marcus (2005) studied the dynamics and formation of vortices in stably stratified protoplanetary disks and found that vortices are unstable under perturbation. Theoretically, the origin of these vortices is understood to be a perturbation in the plane shear flow (UR04; MAN05; AMN05). Continuing in the same line of thought, recently, Umurhan et al. (2006) have shown substantial growth resulting from initial perturbations in the linearly stable steady state and have concluded that significant perturbation-energy amplification occurs in accretion disks on a global scale.

In the present paper we perform a local linear analysis in the shearing-box approximation. Although a small section of an accretion disk may not reproduce the global disk properties, we assume that if turbulence is exhibited at any section, then that sustains and eventually affects the entire disk. While the local result does not guarantee its global signature, the absence of local instability and then possible turbulence, perhaps does guarantee its global absence. Therefore, from the results of this paper we can determine the future avenues of the subject by investigating whether the analyses for secondary instability, including transient growth, are fruitful paths for solving this problem.

In $\S 2$ we outline the basic model considered for describing primary and secondary perturbations. In $\S 3$ we present the simple solution when the evolution of the secondary perturbation is much more rapid than that of the primary one. Subsequently, in $\S 4$ we analyze the general solutions when both perturbations vary simultaneously. Finally we sum up the results with a discussion in $\S 5$.

\section{THE MODEL}

We consider a small portion of the Keplerian flow centered on radius $r_{0}$ (see MAN05; AMN05, for details). Here, the Keplerian flow locally reduces to the rotating Couette flow in a narrow gap limit whose unperturbed velocity vector is $\boldsymbol{U}=(0,-x, 0)$, where $x \ll r_{0}$. Therefore, the Navier-Stokes and the continuity equations for the dynamics of viscous incompressible disk fluid are given by

$$
\frac{\partial \boldsymbol{U}}{\partial t}+\boldsymbol{U} \cdot \nabla \boldsymbol{U}+\boldsymbol{\Omega} \times \boldsymbol{\Omega} \times \hat{\boldsymbol{x}} x+2 \boldsymbol{\Omega} \times \boldsymbol{U}+\nabla(\tilde{p})=\frac{1}{\mathrm{Re}} \nabla^{2} \boldsymbol{U}, \quad \nabla \cdot \boldsymbol{U}=0,
$$

where $\nabla=(\partial / \partial x, \partial / \partial y, \partial / \partial z)$, Re is the Reynolds number, $\boldsymbol{\Omega}=(0,0,1 / q)$ is the angular frequency, $t$ is the time, and $\tilde{p}$ is proportional to the pressure. For a Keplerian disk $q=1.5$, and for a constant angular momentum disk $q=2$.

Here all the variables are expressed in dimensionless units. The unit of length is the box size in the radial direction, and the unit of velocity is the maximum relative velocity between two fluid elements in the box. Below we describe two subsequent perturbation effects, primary and secondary.

\subsection{Primary Perturbation}

Under a linear two-dimensional (primary) perturbation, the velocity vector of the (primary) flow reduces as

$$
\boldsymbol{U} \rightarrow \boldsymbol{U}_{p}=\left(w_{x},-x+w_{y}, 0\right)=\mathbf{A} \cdot \boldsymbol{d},
$$


where

$$
w_{x}=\zeta \frac{k_{y}}{\kappa^{2}} \sin \left(k_{x} x+k_{y} y\right), \quad w_{y}=-\zeta \frac{k_{x}}{\kappa^{2}} \sin \left(k_{x} x+k_{y} y\right),
$$

A is a tensor of rank 2, the position vector $\boldsymbol{d}=(x, y, z), k_{x}, k_{y}$ are the components of the wavevector of perturbation, $\kappa=\left(k_{x}^{2}+k_{y}^{2}\right)^{1 / 2}$, and $\zeta$ is the amplitude of the vorticity perturbation. Now we concentrate on a further small patch of the primarily perturbed flow so that the spatial scale is very small compared to the wavelength of the primary perturbation, $x \ll 1 / k_{x}, y \ll 1 / k_{y}$. Therefore, $\mathbf{A}$ comes out to be

$$
\mathbf{A}=A_{j}^{k}=\left(\begin{array}{ccc}
\zeta \sqrt{\epsilon(1-\epsilon)} & \zeta(1-\epsilon) & 0 \\
-(1+\zeta \epsilon) & -\zeta \sqrt{\epsilon(1-\epsilon)} & 0 \\
0 & 0 & 0
\end{array}\right)
$$

where $\epsilon=\left(k_{x} / \kappa\right)^{2}$. The above A indicates that the flow pattern is a generalized elliptical one compared to that discussed in standard fluid literature (e.g., Bayly 1986; Craik 1989; Kerswell 2002) as an ordinary elliptical flow given by

$$
A_{j}^{k}=\left(\begin{array}{ccc}
0 & 1-\epsilon & 0 \\
-(1+\epsilon) & 0 & 0 \\
0 & 0 & 0
\end{array}\right) .
$$

\subsection{Secondary Perturbation}

The background flow for further perturbation (secondary one) also corresponds to equation (1) except that $\boldsymbol{U}$ is replaced by $\boldsymbol{U}_{p}$. The secondary perturbation modifies components of the velocity in equation (2) and the pressure as $\boldsymbol{U}_{p} \rightarrow \boldsymbol{U}_{p}+\boldsymbol{u}$ and $\tilde{p} \rightarrow \tilde{p}+p$. The perturbation is considered to be of plane-wave type and given by

$$
\left(u_{i}, \tilde{p}\right)=\left[v_{i}(t), p(t)\right] \exp \left[i k_{m}(t) x^{m}\right],
$$

where $k_{m} \gg k_{x}, k_{y}$. The Latin (subscript/superscript) indices run from 1 to 3 such that, e.g., $x^{m} \equiv(x, y, z)$, and thus the background velocity can be written as $U_{p i}=A_{i}^{m} x_{m}$. Therefore, from equations (1), (4), and (6), replacing $\boldsymbol{U}$ by $\boldsymbol{U}_{p}$, and after some algebra, we obtain the evolution of a linear secondary perturbation

$$
\dot{v}_{j}+A_{j}^{k} v_{k}+2 \epsilon_{m k j} \Omega^{m} v^{k}=-i p k_{j}-\frac{v_{j}}{\operatorname{Re}} k^{2},
$$

along with

$$
k_{n} v^{n}=0, \quad \dot{k}_{j}=-\left(A_{j}^{m}\right)^{T} k_{m}, \quad k_{n} \dot{v}^{n}=k_{m} A_{n}^{m} v^{n},
$$

where the "overdot" indicates a derivative with respect to $t, \epsilon_{m k j}$ is a Levi-Civita tensor, and $k^{2}=k_{m} k^{m}$.

Two components, $k_{1}$ and $k_{2}$, of the wavevector $\left[\boldsymbol{k}=k_{m}=\left(k_{1}, k_{2}, k_{3}\right)\right]$ of secondary perturbation oscillate in time with the angular frequency $\varpi=[\zeta(1-\epsilon)]^{1 / 2}$ at a fixed $\epsilon$, while the third one, $k_{3}$, remains constant. As we choose the signature of the background Minkowski spacetime to be $(-,+,+,+)$, it does not matter whether a Latin index appears subscript or superscript. For example, $A_{j}^{k}=A_{j k}$, where $j$ and $k$, respectively, indicate the row and the column number.

Now projecting out equation (7) by $P_{i}^{j}=\delta_{i}^{j}-k^{-2} k^{j} k_{i}$ and using equation (8), we obtain

$$
\dot{v}_{i}=\left(2 \frac{k^{j} k_{i}}{k^{2}}-\delta_{i}^{j}\right) A_{j}^{k} v_{k}-2 \epsilon_{m k i} \Omega^{m} v^{k}-\frac{v_{i}}{\operatorname{Re}} k^{2}+\left(2 \epsilon_{m k j} \Omega^{m} v^{k}+\frac{v_{j}}{\operatorname{Re}} k^{2}\right) \frac{k^{j} k_{i}}{k^{2}} .
$$

A similar equation was obtained by Bayly (1986), with the difference that equation (9) has additional terms induced due to the Coriolis and the viscous effects.

As Re is very large in an accretion disk, we neglect the viscous term in equation (9) after comparing it with others and rewrite the equation as

$$
\dot{v}_{i}=\Lambda_{i}^{j} v_{j}
$$

where

$$
\Lambda_{i j}=\left(\begin{array}{ccc}
\left(\frac{2 k_{1}^{2}}{k^{2}}-1\right) A_{11}+\frac{2 k_{1} k_{2}}{k^{2}}\left(A_{21}+\frac{1}{q}\right) & \frac{2 k_{1}^{2}}{k^{2}}\left(A_{12}-\frac{1}{q}\right)+\frac{2}{q}-A_{12}+\frac{2 k_{1} k_{2}}{k^{2}} A_{22} & 0 \\
\frac{2 k_{1} k_{2}}{k^{2}} A_{11}+\frac{2 k_{2}^{2}}{k^{2}}\left(A_{21}+\frac{1}{q}\right)-A_{21}-\frac{2}{q} & \frac{2 k_{1} k_{2}}{k^{2}}\left(A_{12}-\frac{1}{q}\right)+\left(\frac{2 k_{2}^{2}}{k^{2}}-1\right) A_{22} & 0 \\
\frac{2 k_{1} k_{3}}{k^{2}} A_{11}+\frac{2 k_{2} k_{3}}{k^{2}}\left(A_{21}+\frac{1}{q}\right) & \frac{2 k_{1} k_{3}}{k^{2}}\left(A_{12}-\frac{1}{q}\right)+\frac{2 k_{2} k_{3}}{k^{2}} A_{22} & 0
\end{array}\right)
$$


We essentially need to solve equation (10) to demonstrate the behavior of perturbations. Next we describe the solution in various possible situations.

\section{PERTURBATION SOLUTION AT A FIXED $\epsilon$}

The solution with constant $\epsilon^{2}$ implies the result at a particular instant in the evolution of the primary perturbation that provides the instantaneous growth rate. The underlying idea is to focus on the situation when the evolution of the secondary perturbation and the corresponding development of growth is much more rapid than that due to the primary perturbation, and therefore $\epsilon$ remains practically constant during secondary perturbation evolution. This helps us to compare the result with those from standard fluid literature (e.g., Bayly 1986; Kerswell 2002).

The general solution of equation (10) can be written as a linear superposition of Floquet modes

$$
v_{i}(t)=\exp (\sigma t) f_{i}(\phi)
$$

where $\phi=\varpi t, f_{i}(\phi)$ is a periodic function with time period $T=2 \pi / \varpi$, and $\sigma$ is the Floquet exponent, which is the eigenvalue of the problem. Clearly, if $\sigma$ is positive, then perturbation increases with time. By applying the periodicity condition, $f_{i}(2 \pi)=f_{i}(0)$, to equation (12), we obtain

$$
v_{i}(T)=\exp (\sigma T) f_{i}(2 \pi)=\exp (\sigma T) f_{i}(0)=\exp (\sigma T) v_{i}(0) .
$$

Therefore, $\exp (\sigma T)$ serves as an evolution operator.

To determine the exponential growth rate, $2 \sigma$, we strictly follow Bayly (1986) and Craik (1989). In this method, one has to evaluate the associated velocity evolution matrix, whose eigenvalue and eigenvector at $t=T$ are $e^{\sigma T}$ and $f_{i}(2 \pi)=f_{i}(0)$, respectively, satisfying

$$
\frac{d M_{j i}(t)}{d t}=\Lambda_{j}^{m} M_{m i}(t)
$$

where $M_{j i}(0)=\delta_{j i}$. Essentially $M_{j i}(t)$ serves as an evolution operator such that

$$
v_{j}(t)=M_{j i}(t) v_{i}(0)
$$

Thus, using the fourth-order Runge-Kutta method, one can easily compute the elements of the $3 \times 3$ matrix $M_{j i}(T)$. An interesting feature to note is that $\left\langle\operatorname{Tr}\left(\Lambda_{i j}\right)\right\rangle=0^{3}$ over $0 \leq t \leq T$. Therefore, $\operatorname{det}\left[M_{j i}(T)\right]=1$. Moreover, $d\left[k_{j} M_{i}^{j}(t)\right] / d t=0$, and therefore $k_{i}(0)=$ $k^{j}(0) M_{j i}(T)$, which indicates that one eigenvalue of this $3 \times 3$ matrix is always unity. The remaining two eigenvalues of $M_{j i}(T)$ must be either real and reciprocal to each other or complex conjugate to each other with unit modulus. This property helps us to check the accuracy of our results, because the product of all three eigenvalues must be unity. Therefore, our problem reduces to evaluating two nontrivial eigenvalues, $\mu_{1}, \mu_{2}$, of the matrix $M_{j i}(T)$. If $\mu_{1}$ or $\mu_{2}$ is real and positive, then the Floquet exponent $\sigma_{i}=\log \left(\mu_{i}\right) / T$.

When the initial value of $\boldsymbol{k}=\boldsymbol{k}(0)=k_{m}(0) \equiv(0,0,1), \boldsymbol{k}(t)$ remains conserved throughout as follows from equation (8). This is the pure vertical perturbation. In this case, $\Lambda_{j i}$ in equation (11) appears as a constant matrix. Therefore, $e^{\sigma t}$ and $f_{i}(t)$ are the eigenvalue and the eigenvector, respectively, of the matrix $M_{j i}(t)$ at any time $t$, and thus the Floquet exponent can be evaluated at any instant. In fact, there is an exact analytical solution for the Floquet exponents for this initial condition, which are the eigenvalues of the matrix $\Lambda_{j i}$.

\subsection{Ideal Elliptical Flow}

Before investigating our accretion disk solutions in detail, let us recapitulate the nature of standard elliptical flow and the corresponding instability that has long been discussed in the fluid dynamics literature. The velocity of a two-dimensional fluid element with elliptical streamlines is given by $\boldsymbol{U}_{p}=\mathbf{A} \cdot \boldsymbol{d}$ (see, e.g., Kerswell 2002) with the definition of $\mathbf{A}$ from equation (5).

Now following the perturbation technique described in $\S 2.2$, we obtain $\Lambda_{i j}$ given by equation (11) with the components of $\mathbf{A}$ from equation (5). If $\boldsymbol{k}$ is constant, i.e., perturbation is vertical, then $\Lambda_{i j}$ is a constant matrix given by

$$
\Lambda_{j i}=\left(\begin{array}{ccc}
0 & 2 \Omega_{z}+\epsilon-1 & 0 \\
-2 \Omega_{z}+\epsilon+1 & 0 & 0 \\
0 & 0 & 0
\end{array}\right)
$$

whose nontrivial eigenvalues are the velocity growth rates (Floquet exponents) given by

$$
\sigma= \pm \sqrt{\epsilon^{2}-\left(1-2 \Omega_{z}\right)^{2}}
$$

For a Keplerian flow, $\sigma= \pm\left(\epsilon^{2}-\frac{1}{9}\right)^{1 / 2}$. Therefore, the vertical perturbation gives rise to the positive instantaneous growth rate in a Keplerian disk for $\epsilon>\frac{1}{3}$. For any other perturbation, $k_{1}$ and $k_{2}$ oscillate in time with the angular frequency $\varpi=\left(1-\epsilon^{2}\right)^{1 / 2}$, and $k_{3}$ remains constant (for detailed descriptions, see, e.g., Bayly 1986; Craik 1989; Kerswell 2002). In Figure 1 we compare the variation of the maximum velocity growth rate as a function of the eccentricity parameter for a Keplerian flow with that of a nonrotating flow. By

\footnotetext{
${ }^{2}$ Remember that the radial wavevector of the primary perturbation, $k_{x}$, varies with $t$ (MAN05; AMN05), therefore in reality the background for the secondary perturbation appears to be time dependent.

3 "Tr" denotes the sum of diagonal elements of the matrix, $\langle\ldots\rangle$ indicates the averaged value, and "det" refers to the determinant of the matrix.
} 


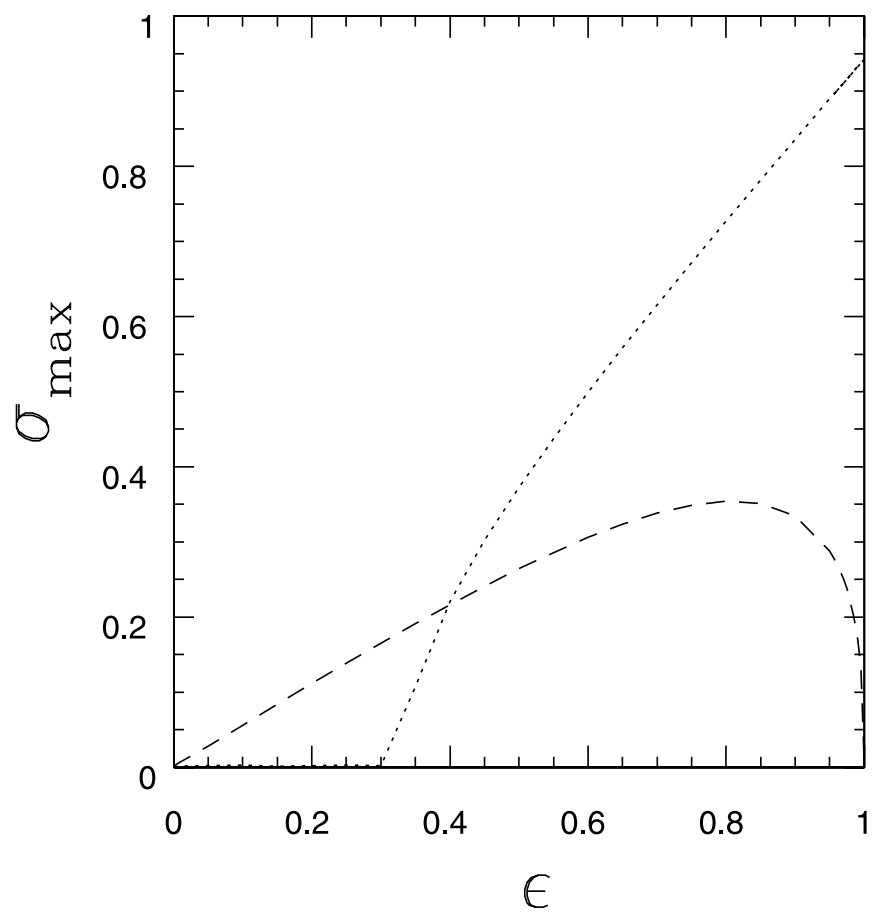

FIG. 1.-Variation of maximized growth rate for elliptical instability as a function of the eccentricity parameter. Dashed and dotted curves correspond to results for nonrotating and rotating shear flows, respectively, and $q=1.5$.

"maximum" we mean the quantity obtained by maximizing over $\boldsymbol{k}$. Clearly the growth rate is significantly large at high $\epsilon$ for a Keplerian system, which is interesting for astrophysical purposes. ${ }^{4}$ This is understood physically from equation (17); when $\Omega_{z}=0, \sigma=0$ at $\epsilon=1$. However, for a rotating flow, $\sigma= \pm 2(q-1)^{1 / 2} / q$ at $\epsilon=1$. This result motivates us to study the elliptical streamline (vortex) effects in an actual Keplerian flow, which governs in accretion disks as follows from equation (4).

\subsection{Flow in a Keplerian Disk}

Here the velocity, $\boldsymbol{U}_{p}$, of the background flow (primarily perturbed flow) is defined according to $\mathbf{A}$, given by equation (4). When does our primarily perturbed Keplerian flow reduce to a conventional flow? (1) When $k_{x} \rightarrow \infty$, i.e., when $\epsilon \rightarrow 1$ and $\zeta \rightarrow 1$, A in equation (4) is the same as that in equation (5). This is the case of an extremely eccentric flow. (2) When $k_{x} \rightarrow \infty$ and $\zeta \rightarrow 0$, A in equation (4) reduces to that of the plane shear flow (MAN05; AMN05). (3) When $k_{x} \rightarrow 0$, i.e., when $\epsilon \rightarrow 0$ and $\zeta \rightarrow 1$, again the form of A in both the equations is the same. This is the case of a circular flow. (4) When $k_{x} \rightarrow 0$ and $\zeta \rightarrow 0$, and $\mathbf{A}$ in equation (4) again reduces to that of plane shear flow (MAN05; AMN05).

Now for a pure vertical perturbation, $\Lambda_{j i}$ in equation (11) reduces to

$$
\Lambda_{j i}=\left(\begin{array}{ccc}
-\zeta \sqrt{\epsilon(1-\epsilon)} & 2 \Omega_{z}-\zeta(1-\epsilon) & 0 \\
-2 \Omega_{z}+\zeta \epsilon+1 & \zeta \sqrt{\epsilon(1-\epsilon)} & 0 \\
0 & 0 & 0
\end{array}\right),
$$

where $\Omega_{z}=1 / q$. As before the above $\Lambda_{j i}$ is a constant matrix, and thus we evaluate the Floquet exponent as

$$
\sigma= \pm \sqrt{\zeta \epsilon-\left(2 \Omega_{z}-1\right)\left(2 \Omega_{z}-\zeta\right)}
$$

Most of the works in fluid literature, so far, have been carried out for nonrotating systems without focusing on a Keplerian flow rigorously. Therefore, equation (19) can be seen as an extension of those results for an actual rotating Keplerian flow.

Now we understand the following points from equation (19):

1. When $\Omega_{3}=0, \sigma=[\zeta(\epsilon-1)]^{1 / 2}$. This verifies that nonrotating two-dimensional plane shear flow is always hydrodynamically stable under a pure vertical perturbation.

2. When $\Omega_{3}=\frac{1}{2}, \sigma=(\zeta \epsilon)^{1 / 2}$. Therefore, the constant angular momentum accretion flow is always hydrodynamically unstable. The energy growth rate of perturbation increases with the strain rate, i.e., the eccentricity of the flow.

3. When $\Omega_{3}=\frac{2}{3}, \sigma=[\zeta \epsilon-(4-3 \zeta) / 9]^{1 / 2}$. Therefore, a Keplerian flow with elliptical streamlines gives rise to unbounded growth, at least in certain time intervals when the growth and growth rate due to the primary perturbation is very small, under a pure vertical perturbation, only if $\zeta>\frac{1}{3}$.

\footnotetext{
${ }^{4}$ Remember that our rotating Keplerian flow described in $\S 2$ is highly eccentric at large $k_{x}$, i.e., at the early stage of the evolution of primary perturbation.
} 


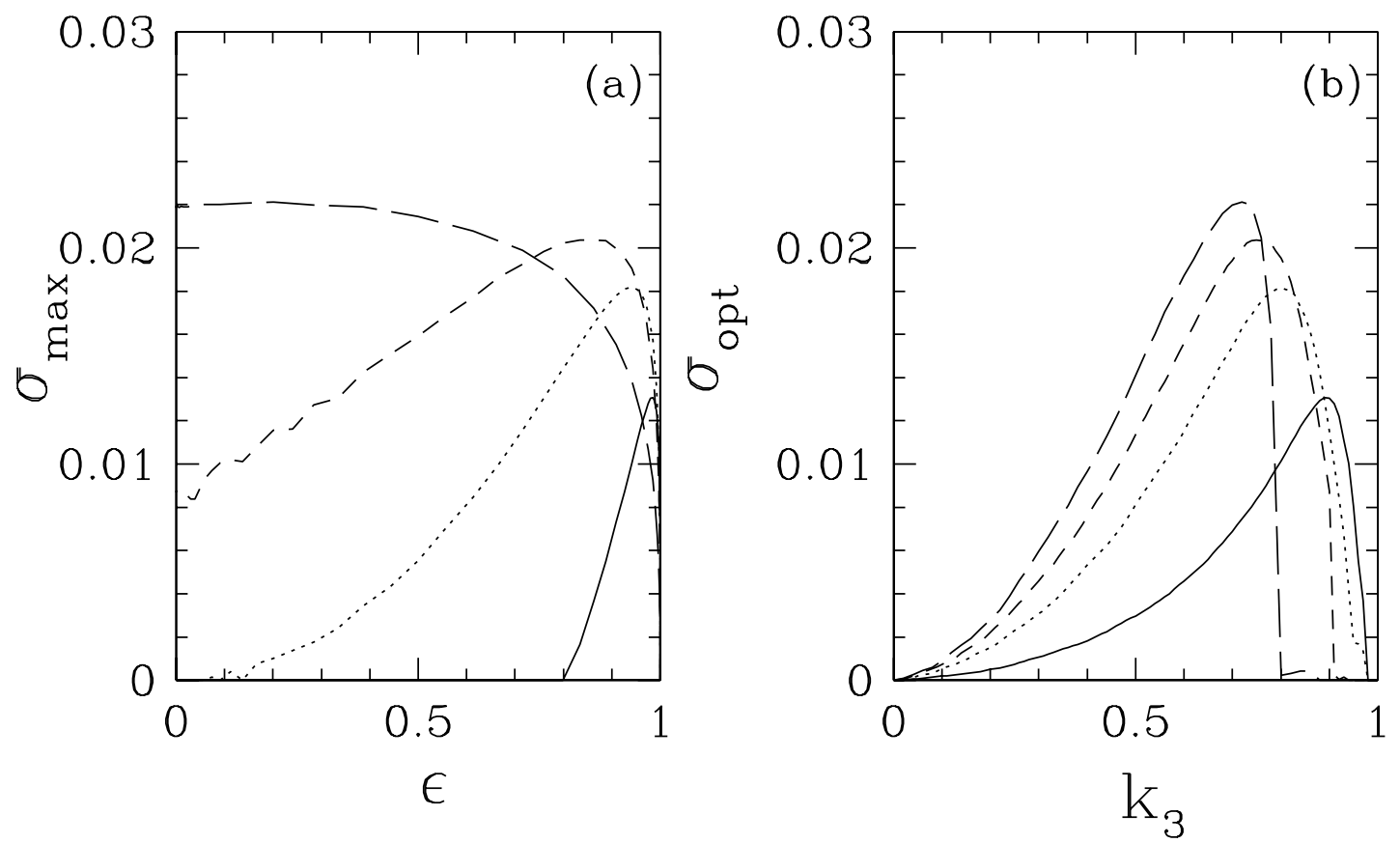

FIG. 2.-(a) Variation of maximum growth rate as a function of the eccentricity parameter. Solid, dotted, dashed, and long-dashed curves indicate the results for $\zeta=0.2$, $0.1,0.05$, and 0.01 , respectively. $(b)$ Variation of optimum growth rate as a function of the vertical component of the perturbation wavevector. The curves are the same as in (a) with $q=1.5$ and $\left|\boldsymbol{k}_{0}\right|=1$ throughout.

However, there are some other three-dimensional perturbations ${ }^{5}$ that can generate a positive growth rate, $\sigma$, in a Keplerian flow with $\zeta<\frac{1}{3}$, which we describe by numerical solutions. As the primary perturbation evolves with time, eccentricity decreases, and then the energy growth rate due to the secondary perturbation changes. Figure $2 a$ shows the variation of the maximum growth rate, $\sigma_{\max }$, as a function of the eccentricity parameter, $\epsilon{ }^{6}$ By "maximum" we refer to the quantity obtained by maximizing over the vertical component of the wavevector, $k_{3}$. Clearly, for $\zeta<\frac{1}{3}$, the growth rate maximizes for three-dimensional perturbations with $k_{3}<1$. At small $\epsilon$ and large $\zeta$, the streamlines of the flow essentially become circular (see eq. [4]), and thus the growth rate severely decreases due to the lack of a significant elliptical vortex. On the other hand, when $\epsilon$ and $\zeta$ both are small, the background structure reduces to plane shear, and therefore any growth arises due to primary perturbation only.

Figure $2 b$ shows the variation of the optimum growth rate, $\sigma_{\mathrm{opt}}$, as a function of $k_{3}$. By "optimum" we refer to the quantity obtained by maximizing over $\epsilon$. An interesting fact to note is that the optimum growth rate is always obtained for a three-dimensional perturbation with a significant vertical component. Moreover, as $\zeta$ increases, the best growth rate is obtained at high $\epsilon$ with large $k_{3}$. Therefore, threedimensional growth is more prompt at larger $\zeta$.

\section{COMPUTATION OF GROWTH WITH SIMULTANEOUS EVOLUTION OF BOTH PERTURBATIONS}

The above results verify that in some parameter range the three-dimensional growth rate due to the secondary perturbation in a rotating shear flow is expected to be real and positive, which motivates us to analyze the simultaneous evolution of both perturbations. As the primary perturbation evolves, $k_{x}$ varies with time and therefore $\epsilon$, and then $\mathbf{A}$ does so. Thus, although equation (8) still remains valid, in general the wavevector of the secondary disturbance is not periodic and the solution of equation (10) cannot be expressed exactly by Floquet modes. Therefore, to compute growth in energy, one has to find out the elements of the energy evolution matrix,

$$
\mathcal{M}_{i k}(t)=M_{i m}(t)^{T} M_{m k}(t),
$$

whose largest eigenvalue is growth in energy at time $t$. As $M_{m k}(t)$ can be obtained from equation (14), computation of $\mathcal{M}_{i k}(t)$ is a trivial job. The actual time variance of $\epsilon(t)$ in equation (14) (and in eq. [11]) is now considered. Clearly, $\mathcal{M}_{i k}(t)$ is the instantaneous energy of the perturbation of the flow, and we recall that $M_{i m}(t)$ is the instantaneous velocity of the perturbation.

Figure 3 depicts the evolution of the best-growing secondary perturbation. It is clear that the perturbation at $t=0$ is a leading wave with a large negative $k_{1}$ (as well as $k_{x}$, although $k_{x} \ll k_{1}$ ), and therefore the flow is highly eccentric at the beginning. With time, $k_{1}$ (as well as $k_{x}$ ) decreases in magnitude and finally becomes zero when growth maximizes at $t=t_{\max }$. With a further increase of time, the wave becomes trailing and growth starts to decrease.

Figure $4 a$ shows that as $\zeta$ increases, the (first) peak value of growth increases, and that it occurs at an earlier time. Comparing with the variation of $k_{1}$ as a function of $t$, as shown in Figure $4 c$, it is very clear that a peak in growth appears when $k_{1}$ approaches zero. As $k_{1}$ in

\footnotetext{
${ }^{5}$ By vertical perturbation we mean that only the vertical component of the initial perturbation wavevector is nonzero, while any other perturbation with a nonzero vertical component of the initial wavevector is called a three-dimensional perturbation.

${ }^{6}$ Note that $\epsilon$ is a parameter that carries the information of the eccentricity of the system but is not the eccentricity itself.
} 

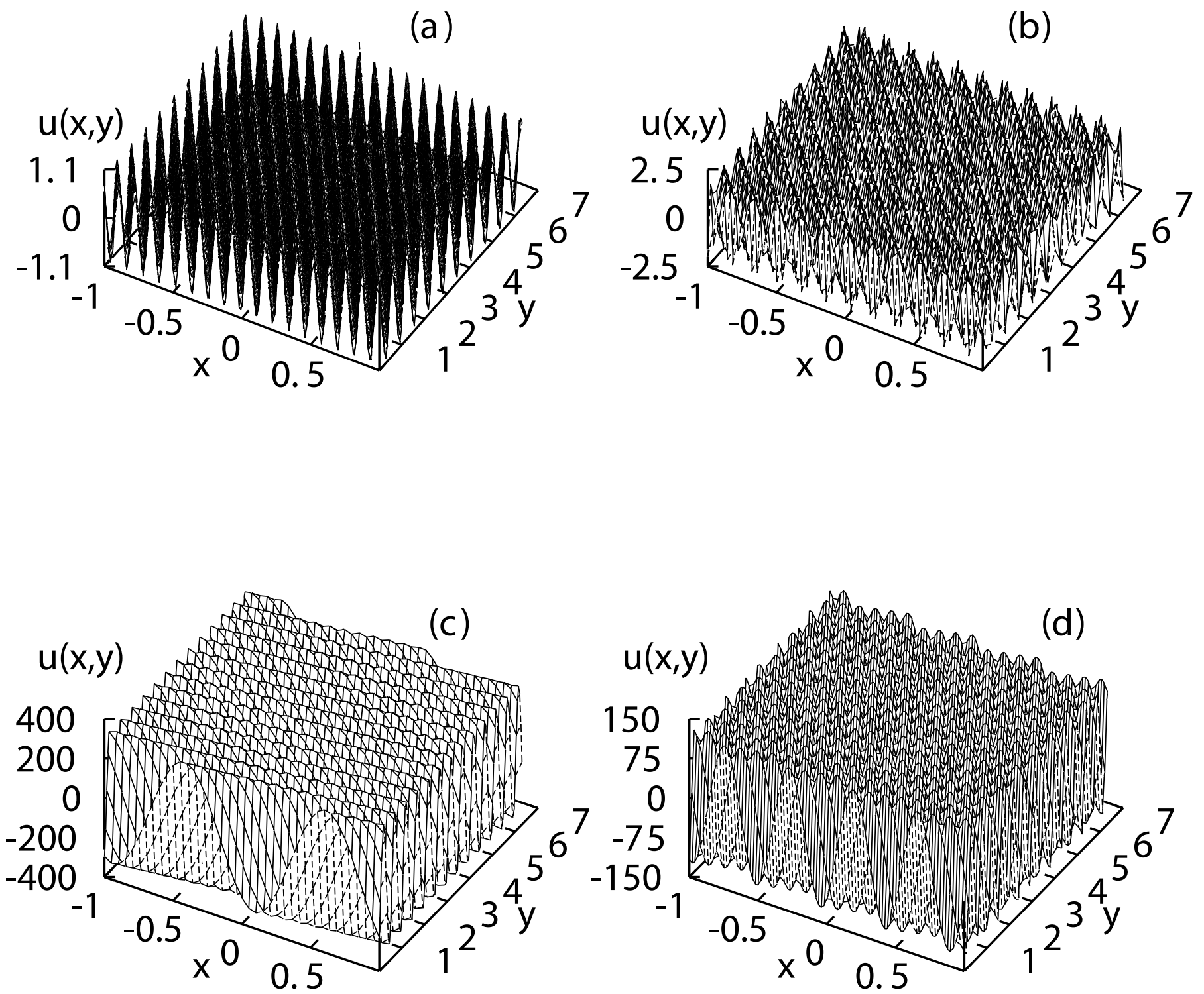

FIg. 3.-Evolution of secondary perturbation in the $x-y$ plane for $\zeta=0.1$, when $(a) t=0$, (b) $t=t_{\max } / 2=386,(c) t=t_{\max }=772$, and $(d) t=3 t_{\max } / 2=1158$. Other parameters are $k_{x 0}=k_{10} / 10=-1000, k_{y}=k_{20} / 10=1, k_{3}=10$, and $q=1.5$. The perturbation is normalized by the magnitude of that at $t=0$.

the cases with $\zeta=0.05$ and 0.1 becomes zero twice, corresponding growth maximizes twice too. The second peak appears at $t \sim 1000$ when $k_{x} \sim k_{1} \rightarrow 0$. Moreover, for $\zeta=0.4, k_{1}$ becomes zero thrice (it crosses the zero line at $t \sim 1000$ and becomes negative, but immediately turns up and crosses the zero again). Therefore, the corresponding growth curve attains two peaks at $t \sim 1000$ very close to each other, apart from the first one at $t=503$. The maximization of growth at the minimization of the radial component of the perturbation wavevector was explained in MAN05 and AMN05. This is essentially due to the fact that $G \propto 1 / k^{2}$. If $\zeta=0$, then $k_{1}$ and $k_{x}$ both become zero simultaneously, as shown in Figure $4 c$. However for $\zeta>0, k_{1}$ increases faster, as follows from equation (8), and becomes zero earlier than $k_{x}$. An interesting fact to note is that underlying growth, although it is apparently of the transient kind as shown in Figure $4 a$, diverges asymptotically for any $\zeta>\frac{1}{3}$ and converges for $\zeta \leq \frac{1}{3}$. The asymptotic divergence of growth is similar to the instability one obtains in linear perturbation analysis for a Poiseuille flow at $\operatorname{Re} \gtrsim 5772$ (e.g., Reddy \& Henningson 1993). The significant asymmetry around $t=t_{\max }$ and the nonzero asymptotic value in growth curves are due to the vertical structure. Figure $4 b$ shows the variation of peak growth as a function $k_{3} / k_{2}$. The quantity $k_{3} / k_{2}$ carries information on how three-dimensional the flow is. It is interesting to note that the maximum growth for $\zeta>\frac{1}{3}$ is unbounded so that it increases with vertical structure, while it is bounded for $\zeta \leq \frac{1}{3}$. We know that in a two-dimensional flow, the maximum growth, $G_{\max }$, scales with $k_{x 0}^{2}$ (MAN05; AMN05). However for $\zeta>0$, $G_{\max }$ decreases at small $k_{3} / k_{2}$ but increases at large $k_{3} / k_{2}$, than that for $\zeta=0$. At around $k_{3} / k_{2}=0.5$, which corresponds to the marginally two-dimensional perturbation, growth due to the secondary perturbation is comparable to that of the primary perturbation. This verifies that the secondary perturbation can govern significant transient growth in a geometrically thin accretion disk with finite vertical thickness. However, in three-dimensions, when $k_{3} / k_{2} \sim 1$, the secondary perturbation effect always dominates over the primary one. This indicates that the three-dimensional secondary perturbation enhances energy growth and thereafter any possible nonlinear feedback effects with its elliptical base state. As $k_{x 0}$ and $k_{10}\left(\sim \mathrm{Re}^{1 / 3}\right.$; see UR04; MAN05; AMN05) increase, the vertical structure plays more effective roles to govern growth. When $k_{x 0}=k_{10} / 10=-10^{3}$ and $k_{y}=k_{20} / 10=1, G_{\max } \sim 4 \times 10^{4}$ at $k_{3} / k_{2}=1$ for $\zeta=0.1$, which is an 

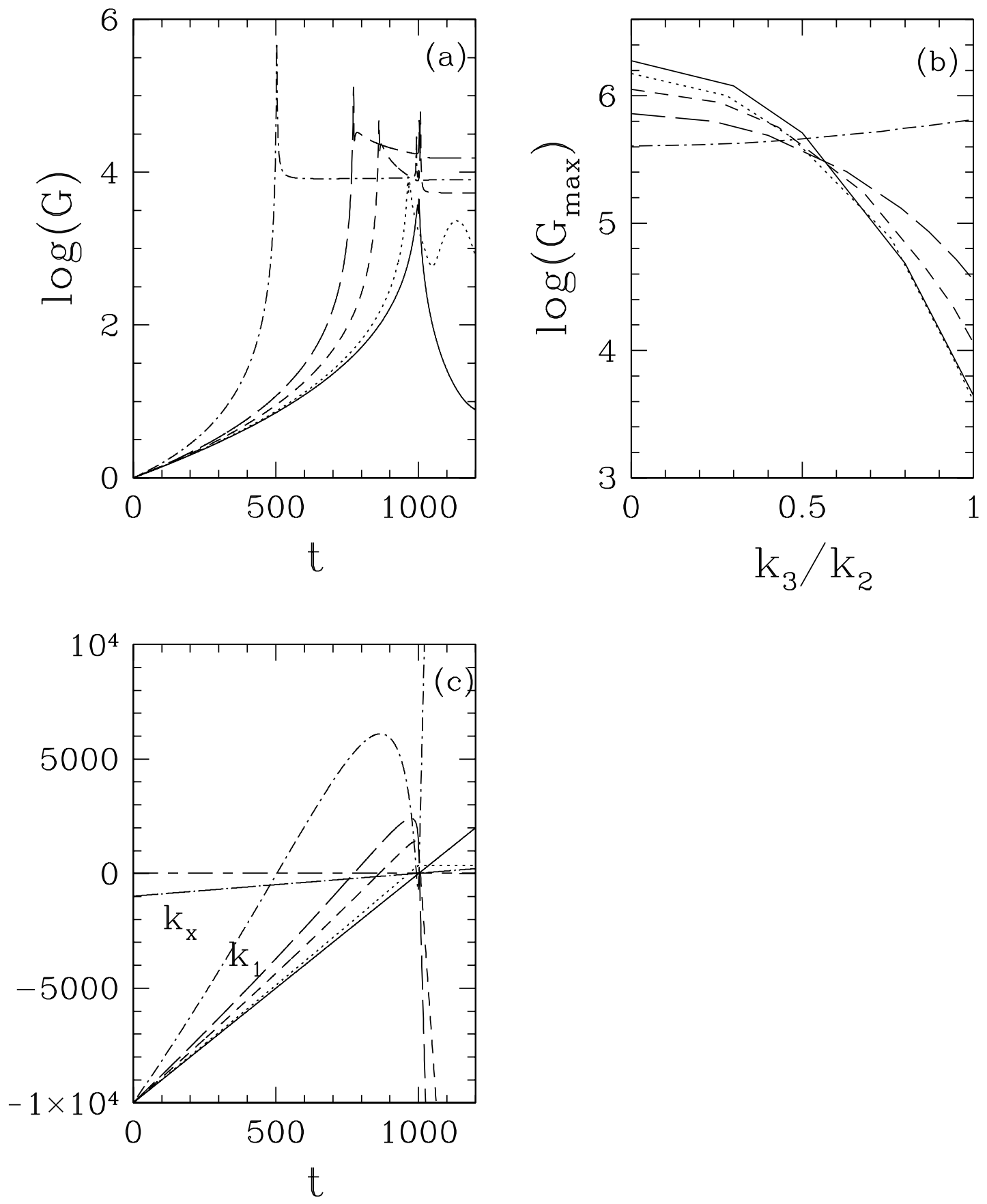

Fig. 4. - (a) Variation of growth as a function of time due to simultaneous evolution of both perturbations when $k_{3}=10$. Solid, dotted, dashed, long-dashed, and dotdashed curves indicate the results for $\zeta=0,0.01,0.05,0.1$, and 0.4 , respectively. (b) Variation of maximum growth as a function of $k_{3} / k_{2}$. The curves are the same as in $(a)$. (c) Variation of radial component of the primary $\left(k_{x}\right.$; dot-long-dashed curve) and secondary $\left[k_{1}\right.$; curves have the same meaning as in $\left.(a)\right]$ perturbation as functions of time of the cases in (a). The short-dash-long-dashed curve indicates the line with a wavevector value of zero. Other parameters are $k_{x 0}=k_{10} / 10=-1000, k_{y}=k_{20} / 10=1$, and $q=1.5$. Note the change in behavior of curves, particularly in $(b)$, when $\zeta>\frac{1}{3}$.

order of magnitude larger than that for $\zeta=0$. If we consider a smaller Re with $k_{x 0}=k_{10} / 10=-10^{2}$, then $G_{\max }$ at $k_{3} / k_{2}=1$ decreases to $\sim 2 \times 10^{3}$ for $\zeta=0.1$, which is still larger by a factor of 2 compared to that for $\zeta=0$. Therefore, three-dimensional effects efficiently enhance growth and then presumably help to trigger turbulence in shear flows.

\section{DISCUSSIONS}

We find that the significant energy growth of the perturbation is possible to govern in shear flow with the Coriolis force. This system is an idealized local analog of an accretion disk, which, under the secondary perturbation, definitely exhibits three-dimensional large growth of the transient kind, and, in addition, sometimes exhibits unbounded late-time growth at a large amplitude of the primary perturbation. We have explicitly demonstrated the perturbation effects one by one. First, a primary two-dimensional perturbation induces a vortex into the flow that can be locally seen as elliptical streamlines. This system, which does not have any exponentially growing eigenmode but does exhibit significant transient growth, has been extensively studied already (e.g., UR04; Yecko 2004; MAN05; 
AMN05). In this situation, a plane-wave perturbation that is frozen into the fluid is sheared along with the background flow. At $t=0$, the effective wavevector of perturbation is in the $x$-direction $\left(k_{x} \gg k_{y}\right)$ and is negative, which provides very asymmetric leading waves. Therefore, the flow at this stage is highly eccentric. As time goes on, the wave fronts are straightened out by the shear, $\left|k_{x}\right|$ decreases, and transient growth increases. When $k_{x} \sim 0$, i.e., when the wave fronts become almost radial, transient growth is at a maximum. At this time, the streamlines of the flow are almost circular. At yet later time, growth decreases and the wave becomes trailing. It has been argued that if the maximum growth exceeds the threshold for inducing turbulence, then this mechanism could drive the system to a turbulent state. Presumably, once the system becomes turbulent, it remains turbulent as a result of nonlinear interactions and feedback among the perturbations. Recall that our present aim is to understand and establish the origin of viscosity in the flow that must be due to turbulence. The transfer of mass inward and angular momentum outward in an accretion disk is difficult to explain in the absence of turbulence. However, the accretion disk is quite a complex system, with possibly continuous perturbed flow. Therefore, if we can address the mechanism that governs turbulence, then its recycling is not difficult.

Second, we consider further perturbation, namely secondary perturbation, in the flow described above. The primarily perturbed shear flow serves as a background for the secondary perturbation whose eccentricity naturally varies with time due to the evolution of the primary perturbation. In this paper we have especially demonstrated the evolution of this secondary perturbation, which exhibits threedimensional large transient growth in a local Keplerian accretion disk. While primary perturbation itself can produce large transient growth at a high Reynolds number, which might drive the nonlinear effects in the system, the best perturbation responsible for this effect is two-dimensional. However, it is understood that perturbations must ultimately decline to zero in the presence of viscosity (see e.g., UR04), unless three-dimensional effects are invoked. Therefore, we have addressed the possible origin of three-dimensional effects, which shows a clear route to three-dimensional hydrodynamic growth and then possible nonlinear feedback and turbulence in accretion flows. Underlying growth arises due to the elliptical vortices present in the background, rather than from the plane shear that exhibited growth under primary perturbation.

In the standard fluid literature, elliptical instability has been widely discussed as a possible route to self-sustained turbulence in linearly perturbed shear flows, as mentioned in $\S 1$. However, the usual emphasis of those investigations is on nonrotating flows. Craik (1989), while he discussed the elliptical instability in rotating shear flows, did not focus on a Keplerian flow, which is of astrophysical interest. Therefore, in the present paper, we have first discussed the growth rate in standard elliptical flows and compared the results for nonrotating flows with that of rotating ones in $\S 3.1$. We have shown that the growth rate in a Keplerian flow with constant elliptical streamlines is significantly larger than that in a nonrotating flow, particularly at high eccentricity.

However, in reality, when a small section of a Keplerian accretion disk is considered under a two-dimensional linear perturbation, the flow governs distorted elliptical streamlines whose structure varies with time. Therefore, the growth rate due to secondary perturbation at a fixed $\epsilon$ (instantaneous growth rate) as described in $\S 3.2$ decreases significantly compared to that in the flow with idealized elliptical streamlines, unless the amplitude of primary perturbation, $\zeta$, is very large. At a $\zeta>\frac{1}{2}$, a pure vertical secondary perturbation produces the best-growing eigenmode. On the other hand, at a $\zeta \leq \frac{1}{3}$, the best-growing eigenmode arises due to other three-dimensional perturbations with a significant, but not solely, vertical effect. Although the instantaneous growth rate appears to be small for a small $\zeta$ (which is of particular interest), at least compared to the case with idealized elliptical streamlines, actual growth that is the result of simultaneous evolution of both perturbations as described in $\S 4$ can be large enough to exhibit nonlinear effects if the time scale for the evolution of perturbation is large. The time for the evolution of perturbation scales with the Reynolds number of the flow as $\mathrm{Re}^{1 / 3}$ (MAN05; AMN05). As perturbation evolves, $k_{x}$ varies from $-\infty$ to 0 , and thus the eccentricity of the flow decreases from 1 to 0 . Most of the important threedimensional growing modes are generated in the high-eccentricity regime when $0.995 \leq \epsilon \leq 1$ and therefore $10 \leq\left|k_{x}\right| \leq \infty$. An important fact to note is that growth maximizes for three-dimensional perturbations with a significant vertical effect.

We therefore conclude with an important caveat. UR04 already showed via two-dimensional simulations that chaotic motions can persist for a time much longer than the timescale needed for linear growth. However, the corresponding vorticity decays unless the vertical structure is there. In the present paper we have shown the existence of three-dimensional perturbation effects and corresponding eigenmodes that govern large energy growth, and then we suggest the possible existence of nonlinear effects and self-sustained hydrodynamic turbulence in accretion disks. Future work will have to verify our suggestion by numerical simulation with proper resolution and possibly also by nonlinear analytic asymptotic methods.

The author is grateful to Ramesh Narayan for suggesting this problem and for extensive discussion and encouragement throughout the course of the work. The author is also thankful to the referee for his/her constructive suggestions that help to improve the presentation of the paper. This work was supported in part by NASA grant NNG 04-GL38G and NSF grant AST 03-07433.

\footnotetext{
REFERENCES

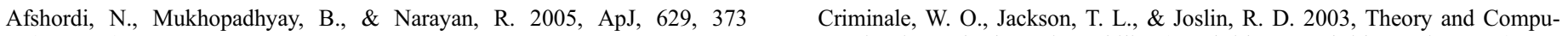
(AMN05)

Balbus, S., \& Hawley, J. 1991, ApJ, 376, 214

Balbus, S., Hawley, J., \& Stone, J. 1996, ApJ, 467, 76

Barranco, J., \& Marcus, P. 2005, ApJ, 623, 1157

Bayly, B. 1986, Phys. Rev. Lett., 57, 2160

Blaes, O., \& Balbus, S. 1994, ApJ, 421, 163

Brosa, U., \& Grossmann, S. 1999, European J. Phys. B, 9, 343

Chagelishvili, G., Zahn, J.-P., Tevzadze, A., \& Lominadze, J. 2003, A\&A, 402, 401

Craik, A. 1989, J. Fluid Mech., 198, 275

Craik, A., \& Criminale, W. 1986, Proc. R. Soc. London A, 406, 13

Criminale, W. O., Jackson, T. L., \& Joslin, R. D. 2003, Theory and Compu-
tation in Hydrodynamic Stability (Cambridge: Cambridge Univ. Press)

Dauchot, O., \& Manneville, P. 1997, J. Phys. II France, 7, 371

Gammie, C., \& Menou, K. 1998, ApJ, 492, L75

Goodman, J. 1993, ApJ, 406, 596

Hawley, J., Balbus, S., \& Winters, W. 1999, ApJ, 518, 394

Hellberg, C., \& Orszag, S. 1988, Phys. Fluids, 31, 6

Ioannou, P. J., \& Kakouris, A. 2001, ApJ, 550, 931

Johnson, B., \& Gammie, C. 2005, ApJ, 626, 978

Kerswell, R. 2002, Ann. Rev. Fluid Mech., 34, 83

. 2005, Nonlinearity, 18, R17

Le Diześ, S., Rossi, M., \& Moffatt, K. 1996, Phys. Fluids, 8, 2084
} 
Lesur, G., \& Longaretti, P.-Y. 2005, A\&A, 444, 25

Longaretti, P.-Y. 2002, ApJ, 576, 587

Lubow, S., Pringle, J., \& Kerswell, R. R. 1993, ApJ, 419, 758

Menou, K. 2000, Science, 288, 2022

Menou, K., \& Quataert, E. 2001, ApJ, 552, 204

Mukhopadhyay, B., Afshordi, N., \& Narayan, R. 2005, ApJ, 629, 383 (MAN05)

Papaloizou, J. C. B. 2005a, A\&A, 432, 743 2005b, A\&A, 432, 757

Pierrehumbert, R. 1986, Phys. Rev. Lett., 57, 2157

Reddy, S. C., \& Henningson, D. S. 1993, J. Fluid Mech., 252, 209

Richard, D., \& Zahn, J.-P. 1999, A\&A, 347, 734

Ryu, D., \& Goodman, J. 1994, ApJ, 422, 269
Schmid, P. J., \& Henningson, D. S. 2001, Stability and Transition in Shear Flows (New York: Springer)

Shakura, N., \& Sunyaev, R. 1973, A\&A, 24, 337

Tevzadze, A., Chagelishvili, G., Zahn, J.-P., Chanishvili, R., \& Lominadze, J. 2003, A\&A, 407, 779

Umurhan, O., Nemirovsky, A., Regev, O., \& Shaviv, G. 2006, A\&A, 446, 1 Umurhan, O., \& Regev, O. 2004, A\&A, 427, 855 (UR04)

Waleffe, F. 1990, Phys. Fluids, 2, 76

$$
\text { 1995, Phys. Fluids, 7, } 3060
$$$$
\text { 1997, Phys. Fluids, 9, } 883
$$$$
\text { 2003, Phys. Fluids, 15, } 1517
$$

Yecko, P. 2004, A\&A, 425, 385 